\title{
Welche analytische Methode zur Bestimmung der sauren Phosphatase (SP) beziehungsweise Prostataphosphatase (PAP) sollte im Routinelabor angewendet werden?
}

\author{
Konkordanz und Diskordanz der Ergebnisse unterschiedlicher Meßprinzipien
}

\author{
G. Oremek ' , U. B. Seiffert ${ }^{1}$, G. Heinert ${ }^{2}$, W. H. Siede ${ }^{1}$, J. Rockenbach ${ }^{2}$ \\ Klinikum der Johann-Wolfgang-Goethe-Universität, Frankfurt \\ 1 Klinisch-Chemisches Zentrallaboratorium, Zentrum der Inneren Medizin \\ 2 Abteilung für Urologie, Zentrum der Chirurgie
}

\section{Zusammenfassung:}

Zur Zeit sind 14 Methoden zur Bestimmung der sauren Phosphatase (SP) bzw. Prostataphosphatase (PAP) mit unterschiedlichen Testprinzipien kommerziell verfügbar. Es sind enzymatische, immunologische, enzymund radio-immunologische Methoden. Es sollte ein Test ausgewählt werden, der klinisch relevante Ergebnisse liefert und eine gute Praktikabilität aufweist. Die enzymatische Bestimmungsmethode der SP nach Hillmann erfüllt beide Kriterien, ebenso wie einige Tests, die monoklonale Antikörper zur Analytik verwenden. Der enzymatische Test verursacht geringeren Aufwand im Vergleich zu immunologischen Methoden. Diesem sollte daher der Vorzug gegeben werden. Keine der Bestimmungsmethoden kommt als sicheres Indiz für eine mögliche Prostataerkrankung speziell als Frühdiagnostikum in Betracht. Die Sensitivitäten betragen maximal $55 \%$.

Die Stabilität der SP im Serum ist problematisch. Enzymaktivitäten bleiben in Gegenwart von Na-hydrogensulfat als Stabilisator konstant, während das Antigen für immunologische Tests beachtlich labil ist. Wir haben innerhalb von 24 Std. bei $+4^{\circ} \mathrm{C}$ durchschnittlich 13,8\% Verlust an immunologisch meßbarer Prostataphosphatase (PAP) beobachtet.

\section{Schlüsselwörter:}

Saure Phosphatase - Prostataphosphatase - Enzymatische Methoden - Immunologische Methoden Stabilität der Proben

\section{Summary:}

At present time 14 different methods, enzymatic, immunologic and enzyme-immunologic, for the determination of acid phosphatase (SP) or prostatic acid phosphatase $(P A P)$ are commercially available. This study was designed to choose a test exhibiting good clinical relevance and practicability. The enzymatic determination of $S P$ according to Hillmann as well as several tests using monoclonal antibodies fulfill both criteria. The Hillmanntest was prefered, because additionally costs and time involved were minimal.

None of these methods is acceptable as a screening test for prostata carcinoma. We measured sensitivity of $55 \%$ in patients suffering from carcinoma.

The stability of $\mathrm{SP}$ in serum is problematic. Enzyme activities can be stabilized by $\mathrm{NaHSO}_{4}$, but the antigen used in immunological tests is labile in vitro. Over a period of $24 \mathrm{~h}$ at $4^{\circ} \mathrm{C}$ a mean decrease of $13.8 \% \mathrm{SP}$ occurred. A stabilizer for the antigen is not known. Frozen samples can be stored without decrease of SP activity for a short period.

Keywords:

Prostatic acid phosphatase - isoenzyme of acid phosphatase - Hillmanntest - stability

\section{Einleitung}

Maligne Neoplasien der Prostata könnten optimal behandelt werden, wenn sie rechtzeitig (d.h. in einem Stadium, in dem sie noch lokal begrenzt sind) diagnostizierbar wären. Unter diesem Aspekt fragt der Arzt nach einem klinisch-chemischen Test, der dazu in der Lage wäre. In die saure Prostataphosphatase (PAP) als Krankheitsindikator hat man solche Erwartungen gesetzt, und deshalb ist PAP Gegenstand und biochemische Grundlage zahlreicher diagnostischer Tests. Neuentwicklungen bringen mehr Spezifität, größere Empfindlichkeit und Verbesserungen. Der Einsatz spezifischer monoklonaler Antikörper hat neue Hoffnungen geweckt. Wir haben 14 Tests zum 
Nachweis von PAP in der Literatur und auf dem Markt gefunden.

Jeder Test wurde bereits klinisch geprüft $(1-7,9,11,12$, 14-16, 20). Leider mangelt es an einer gesamten bzw. einheitlichen Prüfung aller zur Verfügung stehender Tests, um wirklich den Besten herauszufinden. Vergleiche sollten generell mit dem besten und nicht mit dem schwächsten Test als Bezugsgröße erfolgen.

In dieser Publikation werden Ergebnisse von 6 Testmethoden präsentiert. Den Autoren kommt es darauf an, für die Routinediagnostik bezüglich der Sensitivität eine klinisch-relevante Methode aus dem vielfältigen Angebot der Testkombinationen herauszufinden. Die Praktikabilität und die Kosten müssen auch in Betracht gezogen werden. Zusätzlich soll geklärt werden, ob ein Test tatsächlich herausragt und die sichere Diagnostik des Prostata-Karzinoms ermöglicht.

\section{Material, Methoden und Geräte}

Normbereiche zu jeder Methode sind aus der Literatur mehrfach bekannt. Als allgemein gebräuchliche Normwerte betrachten wir die von den Firmen zusammengestellten Werte. Abweichende Normwerte werden mit der zugehörigen Literaturstelle gesondert ausgewiesen (Tab. 2). Spezifitäten sind den Angaben der Firmen entnommen.

\section{Enzymatische Methoden}

Methode 1 erfaßt tartrathemmbare saure Phosphatase (SP) mit p-Nitrophenylphosphat als Substrat (Boehringer Mannheim). Alle Isoenzyme der SP spalten p-Nitrophenylphosphat. Während die PAP durch Tartrat hemmbar ist, werden die SP aus Thrombozyten, Erythrozyten und Knochen nicht gehemmt. Die Hemmung ist aber nicht $100 \%$ ig, so daß die Spezifität zwar erhöht, aber klinisch nicht ausreichend hoch wird $(2,12,15)$.

Methode 2 erfaßt tetrathemmbare SP mit 1 -Naphthylphosphat als Substrat (nach Hillmann, Boehringer Mannheim). Dieses Substrat wird vorwiegend von PAP gespalten, aber auch von der SP aus Thrombozyten und Erythrozyten. Die Kombination mit Tartrat als Inhibitor verbessert die Spezifität erheblich $(2,12,15,20)$.

Außerdem befindet sich ein Test mit Thymolphthaleinphosphat als Substrat auf dem Markt. Dieser Test wurde nicht geprüft (ACA, Dupont).

\section{Immunologischer Farbtest}

Methode 3 erfaßt PAP (Boehringer Mannheim). Es wird ein polyvalenter Antikörper (Kaninchen) verwendet, der mit Komponenten aus Prostatagewebe des Menschen reagiert. Kreuzreaktion mit anderen SP und Protein sind möglich. Hämolyse oder hohe Glukosekonzentration können die Analytik stören (4).

\section{Enzymimmunoassay}

Methode 4 erfaßt PAP mit dem PAP-EIA-Abbott (Abbott). Die Analytik verwendet monoklonale Antikörper, die mit Prostatasekret des Menschen bei der Maus erzeugt werden. Kreuzreaktion mit SP aus anderen Organen ist möglich $(5-7,9,11,14)$.
Methode 5 erfaßt PAP (Immunoassay-Merck) und verwendet monoklonale Antikörper, die mit Prostatasekret des Menschen beim Schaf erzeugt werden. Kreuzreaktion ist möglich auch mit Rheumafaktoren und nach Hämolyse $(10,24)$.

Methode 6 erfaßt PAP (Enzygnost-PAP-Behring Werke) und verwendet polyvalente Antikörper, die mit Prostatasekret des Menschen beim Schaf erzeugt werden. Kreuzreaktion mit SP aus anderen Organen ist möglich (11, 13).

\section{Radio-Immuno-Assays}

Es befinden sich eine Reihe von Tests auf dem Markt von Serono-Diagnostik, Byk Mallinckrodt, Behring-Werke, Abbott, NEN Chemicals, Diagnostic-Products-Corporation und Biermann. Diese Tests haben wir in unsere Untersuchung nicht mit einbezogen, weil wir davon ausgehen, daß die ElAs die gleiche Spezifität wie die RIAs besitzen (16).

\section{Patientenkollektiv}

336 Patienten der urologischen Ambulanz stellen das betrachtete Patientenkollektiv dar. Dem Kollektiv gehören keine gesunden Personen an. Die Prostataerkrankungen wurden durch Echotomographie, rektal-digitale Palpation, Zytologie (Feinnadelbiopsie), histologische Aufarbeitung von Resektionsmaterial sowie Staging-Lymphadenektomie diagnostiziert. Bei allen Patienten mit Karzinomdiagnosen wurde zum weiteren Nachweis von Metastasen eine Ganzkörperskelettszintigraphie und Röntgenuntersuchung durchgeführt. Die Einteilung der Tumore erfolgte nach dem TNM-System (25).

Die klinischen Untersuchungen der Patienten gestatteten eine Einteilung in vier Gruppen mit folgenden gesicherten Diagnosen:

1. Prostata-Adenom $(N=170)$,

2. Prostatitis $(N=42)$,

3. Unbehandeltes Prostata-Karzinom $(N=36)$,

4. Behandeltes Prostata-Karzinom (Hormontherapie und/oder Operation, $\mathrm{N}=88$ ).

Die Seren wurden für die enzymatischen Methoden nach Eintreffen im Labor mit $\mathrm{NaHSO}_{4}(0,36 \mathrm{~mol} / \mathrm{l})$ stabilisiert und sofort untersucht, ein Teil bei $+4^{\circ} \mathrm{C}$, ein weiterer Teil tiefgefroren aufbewahrt. Für die immunologischen Methoden wurden dieselben Seren ohne Zusatz sofort analysiert, ein Teil bei $+4^{\circ} \mathrm{C}$, ein weiterer Teil tiefgefroren aufbewahrt. Die aufbewahrten Proben wurden nach 3 Tagen bzw. nach 14 Tagen erneut analysiert $(N=59)$. Die gefrorenen Proben wurden bei Zimmertemperatur innerhalb von 15 bis 30 min aufgetaut. Die Qualität aller Untersuchungen wurde täglich gesichert.

\section{Geräte}

Methoden 1, 2, 3, 5, 6 wurden mit einem EppendorfPhotometer $1101 \mathrm{M}, \mathrm{Hg} 405 \mathrm{~nm}, 37^{\circ} \mathrm{C}$ und Methode 4 mit dem Abbott-Quantum II, Modul B, Mode 1.6, Filtersatz $492 / 600 \mathrm{~nm}, 25^{\circ} \mathrm{C}$ durchgeführt. Methode 2 wurde zusätzlich an einen ACP-Eppendorf adaptiert.

\section{Statistische Auswertung}

Eine Korrelationsrechnung nach Bartlett erlaubt einen Vergleich der verschiedenen Methoden. Zur Überprüfung von Konkordanz und Diskordanz der Meßwerte mit den' 
Tab. 1: Vergleiche der Stabilität der Enzymaktivität (Methode 2) und des Antigens der PAP (Methode 4) nach 1- und 3tägiger Aufbewahrung bei $+4^{\circ} \mathrm{C}$ (in vitro)

\begin{tabular}{|c|c|c|c|c|c|c|}
\hline \multirow[b]{2}{*}{ Methode } & \multicolumn{2}{|c|}{$\begin{array}{l}\text { Primär- } \\
\text { wert }\end{array}$} & \multicolumn{2}{|c|}{$\begin{array}{l}\text { Nach } \\
1 \text { Tag }\end{array}$} & \multicolumn{2}{|c|}{$\begin{array}{l}\text { Nach } \\
3 \text { Tagen }\end{array}$} \\
\hline & 2 & 4 & 2 & 4 & 2 & 4 \\
\hline $\begin{array}{l}\text { Mittelwert } \\
\text { Verlust \% }\end{array}$ & $\begin{array}{l}6,1 \\
0\end{array}$ & $\begin{array}{l}6,2 \\
0\end{array}$ & $\begin{array}{l}5,8 \\
4,7\end{array}$ & $\begin{array}{r}5,3 \\
13,8\end{array}$ & $\begin{array}{l}5,7 \\
6,8\end{array}$ & $\begin{array}{l}4,3 \\
28\end{array}$ \\
\hline
\end{tabular}

$\mathrm{N}=30$

klinischen Diagnosen wurde die Vierfeldertafel verwendet. Der Auswertung liegt die Publikation von Büttner (8) zugrunde.

\section{Ergebnisse}

Die Stabilität der PAP in den Proben wurde zuerst überprüft. Zu diesem Zweck wurden bewußt Seren ausgewählt, deren Meßwerte über dem Normbereich lagen. Die Proben wurden wie vorher beschrieben behandelt. Tab. 1 zeigt, daß die Enzymaktivitäten (Methode 2) in stabilisierten Seren weitgehend konstant bleiben. Die Aktivität sinkt bei Aufbewahrung bei $+4^{\circ} \mathrm{C}$ durchschnittlich um $6,3 \%$ innerhalb von 3 Tagen. Dagegen ist das Antigen der SP sehr labil (Methode 4). Innerhalb von 24 Std. werden $13,8 \%$ und nach 3 Tagen $28 \%$ weniger PAP gemessen. Nach 14tägigem Lagern bei $-20^{\circ} \mathrm{C}$ zeigen die Aktivitäten der PAP keinen Verlust.

In Tab. 2 sind Kriterien zur Praktikabilität der Methoden zusammengestellt. Die Kosten und der Zeitaufwand der Tests untereinander werden zum Vergleich auf Methode 2 bezogen. Die Werte dieser Methode werden gleich 1 gesetzt, und die Werte der anderen Methoden entsprechend in Relation dazu berechnet. Bei den Kosten wurde für $1=3,17 \mathrm{DM}$ - und bei der Arbeitszeit für $1=5 \mathrm{~min}$ eingesetzt. Die RIAs dürften seitens der Kosten und des Zeitaufwandes wesentlich über den in Tab. 2 angegebe-
Tab. 3: Sensitivitäten der verschiedenen Methoden bei den einzelnen Kollektiven. Die Sensitivität ist errechnet aus der Anzahl der positiven Werte pro Gesamtzahl der Untersuchungen. Die verwendeten Normwerte siehe Tab.2, Spalte 6a

\begin{tabular}{|c|c|c|c|c|c|c|}
\hline \multicolumn{7}{|c|}{ Methoden } \\
\hline 1 & & 2 & 3 & 4 & 5 & 6 \\
\hline \multicolumn{7}{|c|}{ 1. Adenome $(N=170)$} \\
\hline &, 080 & 0,120 & 0,082 & 0,094 & 0,153 & 0,120 \\
\hline \multicolumn{7}{|c|}{ 2. Prostatitis ( $N=42)$} \\
\hline &, 095 & 0,238 & 0,143 & 0,190 & 0,286 & 0,090 \\
\hline \multicolumn{7}{|c|}{ 3. Unbehandelte Prostata-Karzinome $(\mathrm{N}=36)$} \\
\hline & ,333 & 0,556 & 0,389 & 0,500 & 0,444 & 0,611 \\
\hline \multicolumn{7}{|c|}{ 4. Behandelte Prostata-Karzinome $(N=88)$} \\
\hline & 0,023 & 0,068 & 0,068 & 0,068 & 0,114 & 0,091 \\
\hline
\end{tabular}

nen Werten liegen. Außerdem käme die Entsorgung des radioaktiven Materials und der Geräteaufwand noch hinzu.

In Tab. 3 sind die Sensitivitäten unserer Ergebnisse dargestellt. Prostata-Adenome und Prostatitis können nicht erkannt werden. Der Ermittlung der Sensitivität bei unbehandelten Prostata-Karzinomen liegt ein relativ kleines Kollektiv von 36 Patienten zugrunde.

12 Patienten gehören den Stadien $\mathrm{T}_{1-2} \mathrm{~N}_{0} \mathrm{M}_{0}$ und $24 \mathrm{~Pa}$ tienten den Stadien $\mathrm{T}_{3-4} \mathrm{~N}_{0-+} \mathrm{M}_{0-+}$ an: Die ermittelte Sensitivität für die Tests beträgt höchstens $55 \%$. In dem hier vorgestellten Vergleich werden alle Karzinom-Stadien gemeinsam dargestellt, weil in den einzelnen Gruppen keine Unterschiede feststellbar waren (Unspezifität).

Prostatamassage, die unmittelbar vor der Probenahme erfolgt, kann zu erhöhten Meßwerten der PAP im Serum führen (5) und damit eine scheinbare Sensitivität hervorrufen.

Beim Vergleich der Methoden fällt Methode 1 durch geringe Sensitivität und Spezifität heraus. Diese Methode ist für die Diagnostik unbrauchbar.

Tab. 2: Vergleich der Kosteñ (Spalte 2), des Zeitaufwandes (Spalte 3) und der Normbereiche (Spalte 6) der Methoden (Spalte 1 , Beschreibung siehe Text). Kosten und Zeitaufwand sind in Relation zur Methode 2 (Hillmanntest) dargestellt

Es bedeuten: enz = enzymatisch; PNP = p-Nitrophenylphosphat; 1 -NP = 1-Naphthylphosphat; +Tartr = Tartrat als Inhibitor; $A K=$ verwendeter Antikörper; polyv = polyvalent; mono = monoklonal; a) allgemein gebräuchlicher Normwert, b) abweichender Normwert von der Literatur (Spaite 7, siehe auch Methoden)

\begin{tabular}{lllllll}
\hline Methode & $\begin{array}{l}\text { relative } \\
\text { Kosten }\end{array}$ & $\begin{array}{l}\text { relative } \\
\text { Arbeits- } \\
\text { zeit/Test }\end{array}$ & $\begin{array}{l}\text { analytische } \\
\text { Charakte- } \\
\text { ristika }\end{array}$ & Einheit & $\begin{array}{l}\text { Norm- } \\
\text { bereiche }\end{array}$ \\
\hline 1 & 0,27 & 1 & $\begin{array}{l}\text { enz } \\
\text { PNP+Tartr }\end{array}$ & U/I & a) $>4$ ite- \\
ratur \\
b) $>4,3$ \\
2
\end{tabular}


Tab. 4: Vergloich der enzymatischen Methode nach Hillmann mit den anderen Methoden, getestet am Gesamtkollektiv $(N=336)$. Dio Haufigkeiten der enzymotischon (Methode 2, horizontal) sind gegen dic Häufigkeiten der anderen 5 Methoden (senkrecht) in jeder Vierleldertafel aufgetragen

Die verwendeten Normbereichsgrenzen siehe Tab. 2, Spalte 6a, $p$ - Anzahl der Werte > Normwert. $n=$ Anzahl der Werte $\because$ Norniwert

\begin{tabular}{|c|c|c|c|c|c|c|c|c|}
\hline Methoden & 3 & $\cdots$ & 4 & 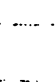 & $\overline{5}$ & & 6 & \\
\hline 230 & 0 & 40 & & 48 & 18 & 46 & & 54 \\
\hline 27430 & 276 & 20 & 276 & 12 & 258 & 14 & 248 & 6 \\
\hline p & $n$ & p & $n$ & $p$ & n & $\mathbf{p}$ & $n$ & $p$ \\
\hline
\end{tabular}

Methode 2 (Test nach Hillmann) weist die gleichen Sensitivitäten und damit fast genauso häufig positive Ergebnisse auf wie die Tests mit monoklonalen Antikörpern. In Tab. 4 sind die Häufigkeiten der positiven bzw. negativen Werte des gesamten Kollektivs dargestellt im Vergleich zur Methode 2 nach Hillmann. In Abb. 1 sind die Meßergebnisse von Methode 2 und 4 quantitativ gegeneinander aufgetragen. Anhand der zugehörigen Normbereichsgrenzen läßt sich erkennen, daß kein einziger falsch-negativer Wert vorkommt und die falsch-positiven Werte bei Methode 2 ausschließlich an der Normbereichsgrenze liegen, wenn die Werte der Methode 4 als Bezugsgröße dienen. Der Korrelationskoeffizient beträgt $r=0,81$.

\section{Diskussion}

Der diagnostische Wert der PAP-Untersuchung wird in der Literatur sehr kontrovers beurteilt. Watson (21) hat aufgrund einer Untersuchung in USA einen predictive value von 0,41 errechnet.

Pfitzenmaier (19) bestätigt, daß $36 \%$ seiner Patienten, die sicher an einem metastasierenden Prostata-Karzinom leiden, keine meßbare Erhöhung der PAP im Serum zeigten. Demnach eignet sich die PAP nicht einmal zur Abgrenzung von Metastasen bei vorliegendem Prostatatumor, geschweige denn zur Frühdiagnostik des ProstataKarzinomes.

Hüting et al. und Keller $(16,17)$ zeigen erneut, daß PAP auch bei gesichertem Prostata-Karzinom selbst mit RIA nicht immer nachweisbar ist.

Schon 1974 berichteten Lung und Yam (18) in einer Zusammenfassung über $\mathrm{SP}$, daß nur spezielle Fälle von Prostataneoplasma mit einer Erhöhung der SP einhergehen. Mehr als $90 \%$ der Fälle im Stadium 1 zeigen keine Veränderung der SP. Sogar in $30 \%$ der Fälle mit Metastasen wird kein positives Ergebnis gemessen. Erst in der Endphase mit zahlreichen Metastasen wird eine Häufigkeit von nicht mehr als $70 \%$ erreicht.

Unsere Ergebnisse weichen in keiner Weise von diesen Berichten ab. Es ragt auch kein Test durch besondere Treffsicherheit heraus. Dieses Ergebnis deckt sich mit bereits früher publizierten Resultaten $(1,3,20)$. Alle Arbeiten unterscheiden sich durch die Auswahlkriterien für die untersuchten Kollektive.

In fast allen Publikationen, die zur Sensitivität der PAP Stellung nehmen, kommt zum Ausdruck, daß ein typi-

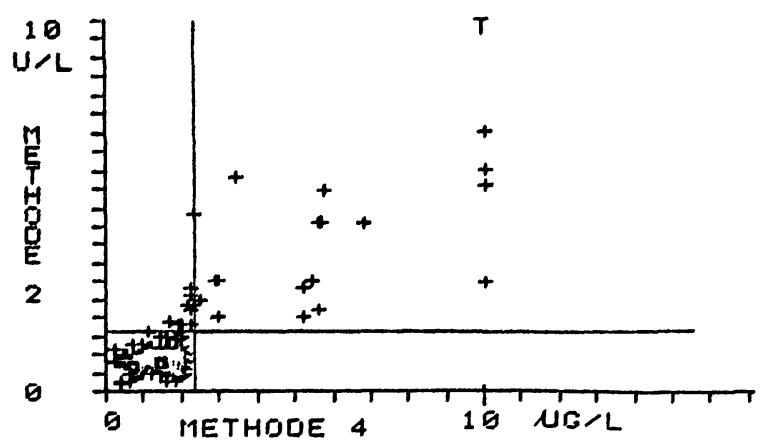

Abb. 1: Vergleich der Meßergebnisse einer immunologischen (Methode 4, Abscisse) und einer enzymatischen Methode (Methode 2 nach Hillmann, Ordinate). Die Werte des Gesamtkollektivs sind gegeneinander oufgetragen. Die eingezeichneten Normbereichsgrenzen trennen normale und pathologische Werte. Nur wenige Werte des Hillmanntests liegen im oberen Grenzbereich bei normalen Werten des immunologischen Testes. Werte $>1.0$ sind bei 10 aufgetragen

sches pathobiochemisches Verhalten der PAP vorliegt, an dem kein Weg vorbeiführt: PAP gelangt bei Prostataerkrankungen und auch Neoplasmen unter Umständen garnicht in das Blut (21). Offensichtlich ist das Wachstum der Karzinomzellen nicht immer mit einer Vermehrung der PAP im Serum verbunden $(17,19)$. Dazu wird festgestellt, daß PAP in der Prostata hauptsächlich in epithelialen Drüsenzellen vorkommt, und diese sind bei Karzinom oft vermindert (18). Aus diesem Umstand resultieren falschnegative Werte in bezug auf die Diagnose.

Eine zweite Unsicherheit kommt hinzu: Die Aktivität der SP im Serum ist die Summe der Aktivitäten von mindestens 5 Isoenzymen (19), die aus verschiedenen Organen stammen. Durch Erhöhung der Spezifität zugunsten der PAP mit pH, Tartrat, Substraten wurde versucht, größere diagnostische Treffsicherheit zu erreichen. Diese Maßnahmen haben zwar eine meßbare Verbesserung der Spezifität gebracht, aber nicht hoch genug, um den Test klinisch sicher zu machen. Aus diesem Umstand resultieren falsch-positive Werte in bezug auf die Diagnose.

Die absolute Spezifität der verwendeten Antikörper ist der Kernpunkt einiger neuer immunologischer Tests. Bisher ist es noch nicht gelungen, einen Antikörper herzustellen, der ausschließlich nur PAP erkennt. PAP und Leukozyten-SP tragen offensichtlich die gleichen Determinanten (23). Auch läßt sich PAP bei Frauen in gleicher Höhe nachweisen wie bei Männern (1).

Zum primären Screening eines Prostata-Karzinoms sind die verfügbaren Testverfahren nicht genügend sicher, denn ein negatives Testergebnis schließt eine Prostataerkrankung nicht aus.

Bauer (3) findet, daß die PAP von allen Zellen der Prostata sezerniert wird und nur das Erscheinen im Serum von sekundären Faktoren verändert ist, so daß das Erkennen erschwert, aber die Diagnostik nicht unmöglich ist.

Es gibt Hinweise, daß eine Erhöhung der SP-Aktivität ein Anzeichen für ein anderes pathophysiologisches Geschehen sein kann (3).

Die berechneten Sensitivitäten sind von Normwerten abhängig. Tab.2, Spalte 6 zeigt, wie unterschiedlich diese in der Literatur angegeben werden. Offensichtlich müßten Werte an einem repräsentativen Kollektiv ermittelt werden. 
Welcher Test sollte klinisch eingesetzt werden?

p-Nitrophenylphosphat als Substrat sollte generell nicht mehr eingesetzt werden. Seiler et al. (20) kamen zu dem Schluß, daß p-Nitrophenylphosphat kein brauchbares Substrat für die PAP-Bestimmung ist wegen geringer Spezifität. Die zusätzliche Verwendung von Inhibitoren hat die Treffsicherheit nur unbedeutend verbessert.

Wir halten Methode 2, den modifizierten Hillmanntest, für einen geeigneten Test im Routinelabor. Wenn auch die Spezifität etwas geringer sein dürfte als bei Methode 4, so sind doch beim Vergleich beider Methoden kein falschnegativer Wert und nur 12 falsch-positive Werte, die ausschließlich an der Normbereichsgrenze lagen, aus 336 Werten aufgetreten. Positive Werte des enzymatischen Testes wird man in Zweifelsfällen mit einem immunologischen Test überprüfen müssen. Die Sensitivität des Hillmanntests ist gleich groß wie die der immunologischen Tests.

Weitere Vorteile können wir gegenüber anderen Tests aufführen. Der Hillmanntest kann auf einem Laborautomaten durchgeführt werden und eignet sich für Analysenserien. Dagegen ist eine Automatisierung bei immunologischen Methoden wegen der Waschprozesse mit gängigen Geräten nicht möglich. Der Preis des Hillmanntests liegt weit unter dem Preis der immunologischen Teste. Die vielen Arbeitsschritte bei den immunologischen Tests und die Stabilität der Enzymaktivität in den Proben zeigen die Vorteile des Hillmanntests.

Stabilität der PAP im Probenmaterial ist eine Voraussetzung für die Brauchbarkeit eines klinisch-chemischen Tests. Die Enzymaktivität der PAP im Serum ist im sauren Milieu für 24 Std. stabil. Dieser Befund findet in der Literatur Bestätigung (22). Bei immunologischen Bestimmungen ist das nicht der Fall. Offensichtlich kommt es in vitro zu einem Verlust an Antigen am PAP-Molekül. Für Proben, die über den Postversand zum Labor gelangen, kommt daher nur ein enzymatischer Test in Frage, solange die Stabilität für immunologische Tests nicht gesichert ist. Wegen der einfacheren Probevorbereitung wurde gelegentlich den immunologischen Tests der Vorzug gegeben. Die Aktivität der SP bzw. PAP ändert sich nicht nach Lagerung in gefrorenem Zustand.

Therapiekontrolle (17) gelingt zwar nicht in allen Fällen, die PAP-Bestimmung ist aber eine wertvolle Hilfe zur Überwachung der Behandlung eines Prostata-Karzinoms.
Schrifttum:

1. ALLNER, R.: Zur Bestimmung der sauren Prostataphosphatase - Ein Methodenvergleich. Lab. med 9,82 (1985).

2. ANDERSCH. M.: Amer. J. Clin. Path. 17, 571-576 (1947), zitiert aus Methoden der enzymatischen Analyse, (Hrsg.) Bergmeyer, H. U. 888-892 (1974) Verlag Chemie Weinheim.

3. BAUER, H. W., STAMMEL, A., TAUBER, R., STURM, W.: Die immunchemische Bestimmung der prostata-spezifischen sauren Phosphatase (PAP) unter Verwendung monoklonaler Antikörper. Tumor Diag. Therapie 5, 29-34 (1984).

4. BAUER, H. W., HACKENBERG, H. J.: Ein neuer Festphasen-Immunoassay für prostata-spezifische saure Phosphatase. Tumor-Diagnostik 3, 138-142 (1982a). 5. BAUER, H. W., WALTER, V., SCHMIEDT, F.: Bestimmung der sauren Phosphatase beim Prostata-Karzinom. Fortschritt Med. 98, 1301-1305 (1980).

6. BAUER, H. W.: Immunenzymatischer Assay für die prostata-spezifische saure Phosphatase beim Prostatakarzinom. Med. Welt 33, 1616-1631 (1982).

7. BAUER, H. W., SCHÜLLER, I., SCHMIEDT, E., DATI, F.: Diagnostik und Verlautskontrolle des Prostata-Karzinoms mit einem Enzymimmunoassay für die prostataspezifische saure Phosphatase. Helv. Chim. Acta 48, 459-463 (1981).

8.. BÜTTNER, J.: Die Beurteilung des diagnostischen Wertes klinisch-chemischer Untersuchungen. J. Clin. Chem. Clin. Biochem. 15, 1 (1977).

9. CHOE. B. K., PONTES, M. K., MORRISON, M. K., ROSE, N. R.: Immunochemical Studies of Prostatic-Acid-Phosphatase. Cancer Treatment Reports 61, 201-204 (1977)

10. CHOE, B. K., PONTES, E. J., DONG, M. K., ROSE, N. R.: Double-Antibody Isoenzyme Assay for Human Prostatic Acid Phosphatase. Clin. Chem. 26, 1854 (1980) 11. COOPER, E. H., BAUER, H. W., DATI, F.: Die immunchemische Bestimmung de sauren Prostata-Phosphatase (PAP) bei Prostata-Karzinomen. Laboratoriumsblätter 32. 87-107 (1982).

12. FISHMAN, W. H., LERNER, F.: J. Biol. Chem. 200, 89-97 (1953) zitiert aus Methoden der enzymatischen Analyse, (Hrsg.) Bergmeyer, H. U. 888-892 (1974) Verlag Chemie Weinheim.

13. FOTI, A. G., HERSCHMANN, H. H., COOPER, J. F.: A solid phase radioimmunoassay for human prostatic acid phosphatase. Cancer Res. 35, 2446 (1975).

14. GRENNER, G., SCHMIDTBERGER, R.: Enzymimmunologische Bestimmung der sauren Prostata-Phosphatase. J. Clin. Chem. Clin. Biochem. 17, 156 (1979)

15. HILLMANN, G.: Fortlaufende photometrische Messung der sauren Prostataphosphatase-Aktivität. J. Clin. Chem. Clin. Biochem. 9, 273 (1971).

16. HÜTING, J., GUNDLACH, G., BLEYL. H.: Diagnostik des Prostata-Karzinoms. Med. Welt 35, 1486 (1984).

17. KELLER, H. E.: Methodik und diagnostische Bedeutung der sauren ProstataPhosphatase. GIT-Verlag, Darmstadt 1983.

18. LUNG, T., YAM, M. D.: Clinical Significance of the Human Acid Phosphatase. Am. J. Med. 56, 604 (1974).

19. PFITZENMAIER, N., JANSSEN, B., MAURER, C., KREMPIEN, B., RIEDASCH G.: Beckenkammbiopsie und Knochenmarkphosphatase zu frühzeitiger Evaluation von Knochenmarkmetastasen bei Prostatakarzinomen. Therapiewoche 28, 1970 (1978). 20. SEILER, D., NAGEL, D.: Saure Phosphatase im Serum (1-Naphthylphosphat). 20. SEILER, D., NAGEL, D.: Saure Phosphatase im Serum (1-Naphthylphosphat).
Referenzwerte und diagnostische Aussage. J. Clin. Chem. Clin. Biochem. 21, 519 (1983).

21. WATSON, R. A., TANG, T.: The predictive value of prostatic phosphatase as a screening test of prostatic cancer. New Engl. J. Med. 303, 497-499 (1980).

22. WEN CHEN, SPERLING, M. H. MAXON, H. R. KAPLAN, L. A.: Stability of Immunologic Activity of Human Prostatic Acid Phosphatase in Serum. Clin. Chem. 28, 1163 (1982).

23. WILLIAM, K. W., LAM, L. T., YAM, M. D., HARRY, J. H.: Comparison of Acid Phosphatase Isoenzyme of Human Seminal Fluid, Prostate and Leukocytes. Clin. Chem. 25, 1285 (1979).

24. YAM L T LI C. Y LAM, K. W. Male Acessory Sex Glands, edited by E. SpringerMilles Hafez, North Holland, Biomedical Press 1980, pp. 183-186.

25. ZIEGENBEIN, R.: Tumor Marker, Gustav Fischer Verlag. Weinheim, 1982.

Anschrift des Verfassers:

Dr. Gerhard Oremek

Klinikum der Johann Wolfgang Goethe-Universität Frankfurt

Klinisch-Chemisches Zentrallaboratorium

Theodor-Stern-Kai 7, 6000 Frankfurt am Main 